\title{
The Precision Optical CAlibration Module for IceCube-Gen2: First Prototype
}

\author{
The IceCube-Gen2 Collaboration ${ }^{\dagger}$ \\ ${ }^{\dagger}$ http://icecube.wisc.edu/collaboration/authors/icrc17_gen2 \\ E-mail: kai.kringsetum.de, elisa.resconi@tum.de, \\ rongen@physik.rwth-aachen. de
}

IceCube-Gen2 is under design, including a Phase-1 extension dedicated to the precision study of atmospheric neutrinos and to an improved search for neutrino astrophysical sources. A new level of precision is needed in order to achieve improved performance with respect to IceCube. A complementary calibration system with respect to the one presently installed in IceCube will enable a better understanding of the ice and will therefore significantly reduce systematic effects. We present a novel specialized calibration device named as the Precision Optical Calibration Module (POCAM). The design of the POCAM is based on the principle of an inverted integrating sphere. An appropriately placed matrix of LEDs in combination with a diffusing layer on the inside of the sphere results in a nearly homogeneous light emission. The output of the LEDs is monitored in-situ to high precision by photosensors, ensuring control over the light output. A first complete prototype has been recently developed and deployed within the cubic kilometer scale Gigaton Volume Detector (Baikal-GVD).

\author{
Corresponding authors: Kai Krings ${ }^{* 1}$, Elisa Resconi ${ }^{1}$, Martin Rongen ${ }^{2}$ \\ ${ }^{1}$ Technische Universität München, Physik-Department, James-Frank-Str. 1, D-85748 Garching \\ bei München, Germany \\ ${ }^{1}$ RWTH Aachen University, III. Physikalisches Institut, Otto-Blumenthal-Str., D-52074 Aachen, \\ Germany
}

35th International Cosmic Ray Conference - ICRC2017

10-20 July, 2017

Bexco, Busan, Korea

${ }^{*}$ Speaker. 


\section{The Precision Optical Calibration Module}

The Precision Optical CAlibration Module (POCAM) is a novel specialized device dedicated to in-situ calibration of the IceCube-Gen2 detector. The goal is to address the primary experimental systematic uncertainties caused by a partial understanding of the optical properties of the ice [1] and the efficiency and angular acceptance of the IceCube Digital Optical Modules (DOMs) [2].

Presently, the systematic uncertainty in IceCube coming from the ice properties is at the level of $10 \%$, being the dominant systematic for a number of analysis. The goal of the POCAM is to reduce these uncertainties down to a level of few \%. Moreover, the POCAM will be a complementary calibration device with respect to other calibration devices, most notably the LEDs already present in the IceCube DOMs.

The POCAM is based on an isotropic, multi-wavelength, and pulsed light source [3, 4]. The POCAM design is shown in Fig. 1. It consists of two glass hemispheres connected by a cylindrical pressure and temperature-resistant titanium (or alternatively stainless steel) housing. Light from a multi-wavelength array of LEDs is driven by a Kapustinsky-style circuit [5] to obtain light pulses of $\sim 10 \mathrm{~ns}$ and $10^{8}-10^{10}$ photons. The pulses are diffused by a sphere in each hemisphere to generate an isotropic and homogeneous ice illumination. The goal is for the light from the hemispheres to be isotropic to within $2 \%$ and the total light output to be determined to within $2 \%$ by in-situ photosensors.

A first complete prototype has recently been deployed within the Gigaton Volume Detector (BaikalGVD), which is under construction in Lake Baikal (Siberia) [6].
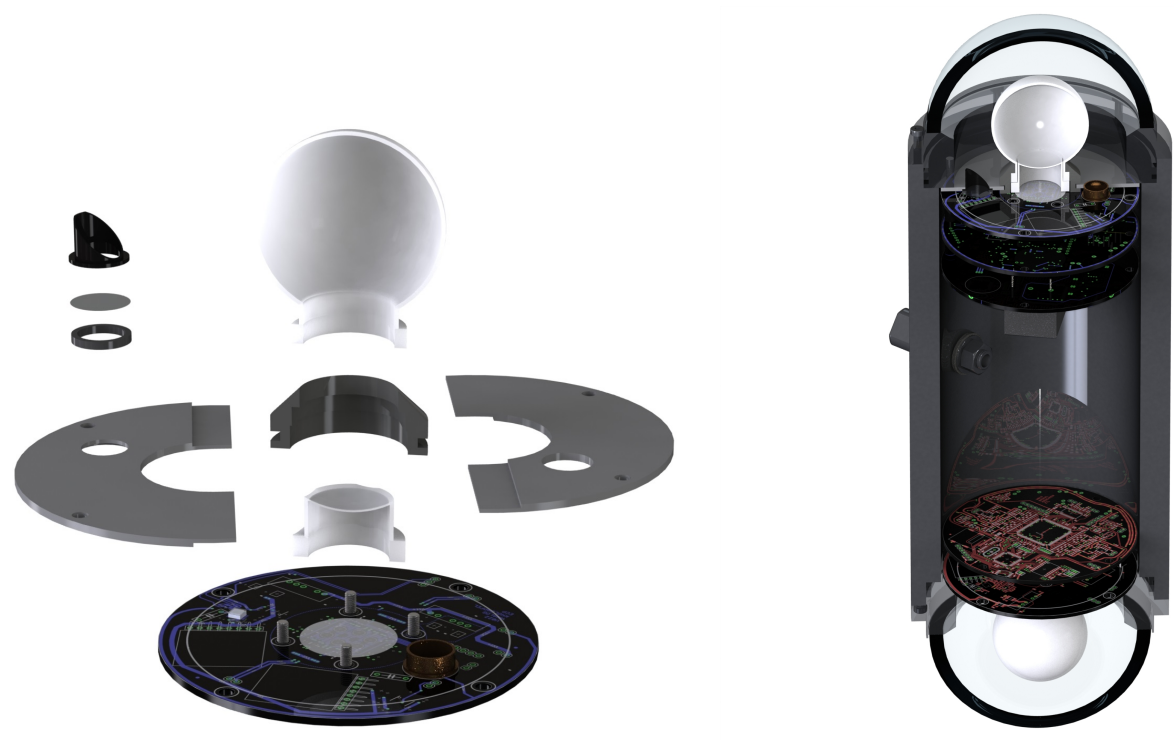

Figure 1: POCAM principle components (left) and complete assembly (right). The POCAM is composed of four sub-systems: the pressure housing, the digital, the analog circuit boards, and the light diffuser elements. Each element is described in more detail in the text. 


\section{The first POCAM prototype}

The POCAM is composed of four sub-systems: the pressure housing, the digital and the analog circuit boards, and the light diffuser elements.

The pressure housing consists of a $15 \mathrm{~mm}$ thick titanium cylinder, the two open sides of which are each closed by a flange and a glass hemisphere attached to it. The glass hemispheres made of BK7 glass have a thickness of $7 \mathrm{~mm}$ and a diameter of 4.5 inches. The entire housing was manufactured by the Nautilus Marine Service $\mathrm{GmbH}$ and specified to a pressure resistance of at least 1500 bar. This corresponds to a $15 \mathrm{~km}$ water column and thus the approximately 10 -fold pressure at the destinations in Antarctica and in Lake Baikal. In fact, larger pressures may arise during the re-freezing process in the Antarctic ice.

An analogue and a digital circuit board are installed within the pressure housing and secured to the titanium flange. They form a fully functional unit that can communicate, emit and register light. Two of these self-sufficient units are installed within the POCAM sharing a common voltage supply board.

A micro-controller, an FPGA, memory chips, an ADC (analog-to-digital converter) and an FPGAbased LED controller are located on each digital board. The micro-controller is responsible for communication and management of the data to be stored on the memory chips. The FPGA takes over all timing sensitive tasks. This includes control signals for the analog board, reading and buffering of the ADC data and controlling the two separate FPGA LED drivers. The two channel $\mathrm{ADC}$ is needed to digitize the signals from the two light sensors. The analog board consists of a light emitting and a light measuring part. The part responsible for the light measurement consists of two different light sensors which are read out via the ADC with the aid of two Cremat chargesensitive rectifiers ${ }^{1}$. Two photosensors are installed in each hemisphere to monitor the emitted light pulse. The one presently installed are a $9 \mathrm{~mm}^{2}$ SiPM (PM3325-EB) from KETEK and a $33 \mathrm{~mm}^{2}$ PIN-Diode from First Light Sensor (PS33-6b-TO). The PIN-Diode has blue-green enhanced efficiency. The PM3325-EB is optimized for blue light and has a low dark count rate and low cross talk probability. To avoid saturation of the SiPM a special filter foil is added. Furthermore, there are additional sensors for environmental monitoring (e.g. temperature, pressure and humidity) on all boards. The light emitting part consists of four Kapustinskis LED drivers with two different pulse configurations (5-10 ns, 15-25 ns) and two colors (blue 455-470 nm, green 525-527 nm) as well as two additional FPGA driven LEDs with longer pulse durations (20 - $80 \mathrm{~ns})$ and higher intensities for a total of three blue and three green LEDs.

Crucial parts of the POCAM are the two diffusing spheres installed on top of the LED arrays. Their task is to convert the strongly anisotropic light from the LEDs into an isotropic diffuse emission profile. For this purpose a CNC-milled hollow PTFE sphere with a wall thickness of $1 \mathrm{~mm}$ and a diameter of $50 \mathrm{~mm}$ has been designed and developed at TU Munich. The LEDs are coupled to the sphere via a PTFE "plug" of 0.5 mm thickness. A first isotropy measurement is shown in Fig. 2.

\footnotetext{
${ }^{1}$ CR-110, CR-113 and operational amplifiers (T.I. LM6171A)
} 


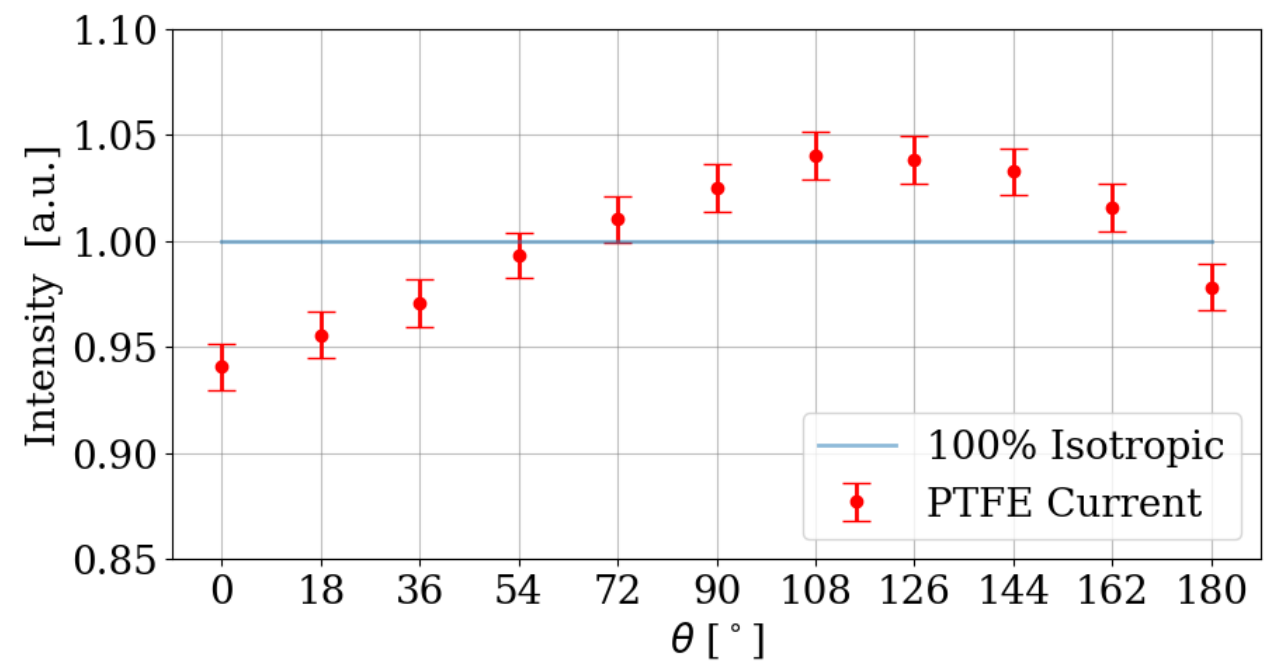

Figure 2: POCAM integrating sphere isotropy measurements. Shown is the angular spectrum with $\theta=$ $90^{\circ}$ being the vertical top of the sphere; the intensity is plotted such that the mean of each measurement corresponds to an intensity of 1.0. It is worth noting that the shift in symmetry towards $108^{\circ}$ is most likely caused by minor alignment and centering difficulties within the measurement setup. Further possibilities, to improve the isotropy and its measurement, are currently being investigated.

The isotropy measurement itself is carried out with a mechanical two-axis rotation device to measure the field of view intensity for various angles; for now this is limited to the polar angle. The SiPM, used as the light sensor is the same that is mounted on the POCAM PCB. However, with its high sensitivity, the components have to be perfectly aligned to result in a symmetric spectrum. An improved and more precise measurement setup is being realized reducing some systematic uncertainties still present in our current measurement setup.

\section{Test Deployment in Baikal-GVD and First Light}

In order to validate the POCAM under realistic conditions, a collaboration with Baikal-GVD has been established [7][8]. In winter 2016-2017, the first POCAM prototype was completed and shipped to Siberia. After a few days of laboratory integration and functional testing, the POCAM has been integrated within GVD on one of the outer strings at depth of $1.100 \mathrm{~m}$. Data analysis of the POCAM-GVD is on-going and will be reported elsewhere.

Early May 2017, detector time in GVD was reserved for POCAM tests and several flasher runs have been carried out. A series of flasher runs have been done at various light intensities: for 100.000 flashes at $53 \mathrm{~Hz}$ the GVD detector response (i.e. the live detector rate) increased from the usual background of 48-49 Hz to around $100 \mathrm{~Hz}$. Fig. 4 shows exemplary data of in-situ SiPM signals. The analysis of the POCAM-GVD data is on-going and will be reported in the future. 


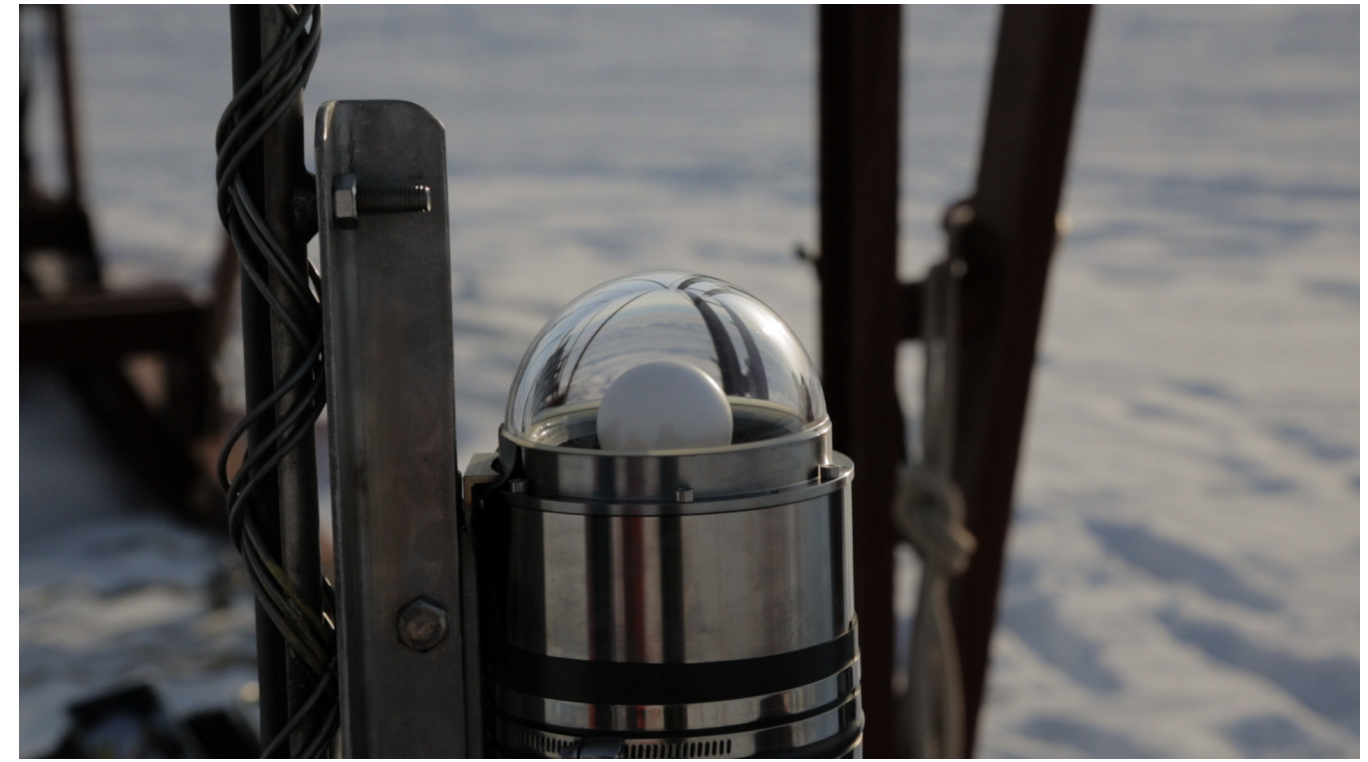

Figure 3: The POCAM module mounted to the GVD string before deployment in the Baikal lake.

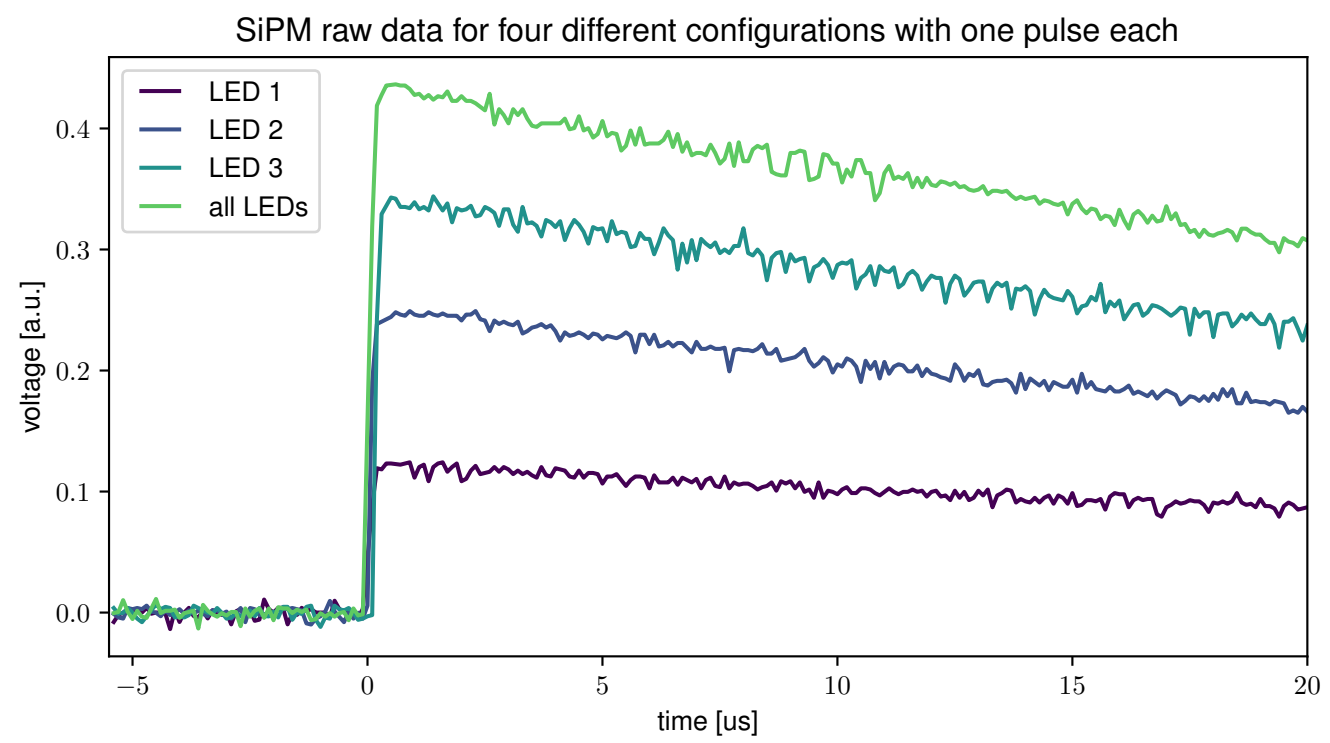

Figure 4: SiPM raw data obtained from POCAM flashes within GVD. LED 1 is the blue, short pulse Kapustinsky, LED 2 is the blue long pulse Kapustinsky, LED 3 is the blue FPGA LED at 40 ns pulse length; all LEDs is the simultaneous flash of a $10 \mathrm{~ns}$ blue FPGA pulse together with 1 and 2.

\section{Anticipated measurements in IceCube-Gen2}

While the POCAM will be a general purpose calibration instrument for IceCube, its isotropic light emissions pattern has clear advantages over the currently used LEDs for a number of specific measurements, which are summarized in the following. 
The glacial Antarctic ice shows an anisotropic scattering amplitude [9], which appears to be aligned with the ice flow direction. With an isotropic light source the systematic uncertainty of the measurement of this effect can be improved as no initial light-emission pattern needs to be assumed. With respect to the detector characteristics the isotropic light pattern enables a direct measurement of the individual relative DOM efficiencies. While the average detection efficiency of all DOMs is well constrained, individual DOMs may deviate from this value by roughly $10 \%$ due to fluctuations in the PMT quantum efficiencies and local ice effects. Currently this measurement is done using cosmic ray induced muon events. It is limited by systematic uncertainties of the selection efficiency of single minimum-ionizing muons.

The POCAM can mimic the light signature of high energy cascades through its calibrated light output. This allows to test the reconstructed cascade energy scale to the uncertainty at which one knows the total POCAM photon output. In addition, by precisely timing the light flashes, closely separated cascades can be mimicked and the cascade vertex separation power, as required for the identification of tau neutrino interactions, can be tested.

A unique possibility measuring the optical properties of the refrozen drill holes will be outlined in more detail in the following.

After the propagation of Cherenkov photons through the bulk glacial ice, each photon detected by a DOM has to also propagate through the refrozen water of the drill holes, called "hole ice". The optical properties of the hole ice are less well understood compared to the bulk glacial ice and are one of the largest uncertainties to neutrino oscillation measurements in IceCube [10]. The hole ice was directly imaged by a camera installed at the bottom of a drill hole. A clear outer region and a central column of $\sim 8 \mathrm{~cm}$ diameter with a very small scattering length (see Fig. 5a) are seen.

The impact of the hole ice is currently being modeled as a modification to the DOMs angular acceptance curve (see Fig. 5b). Here the size of the column determines the relative acceptance in the very forward region $(\cos (\theta)=1)$, while the scattering length modifies the shape around the maximum. Measurements are difficult as the forward region can not be directly probed with the installed LEDs or with Cherenkov light from atmospheric muons. Different measurements and models are currently being used and their range reflects the current uncertainty with respect to the angular acceptance.

To investigate the possibility to improve the in-situ angular acceptance curve measurement using the POCAM, a photon tracking simulation using standard IceCube tools has been performed. A single POCAM is simulated as a point-like and perfectly isotropic emitter situated in the center of the IceCube infill array, DeepCore [11]. Due to expected improvements in the drilling technology, it is assumed that the hole ice surrounding the POCAM has the same properties as the bulk ice, unlike the older IceCube holes. As an initial simplification the bulk glacial ice is assumed to be free of scattering. The same study can be performed in realistic bulk ice, by applying stringent timing cuts on the photon propagation delay from the POCAM to each receiving DOM, ensuring a straight, unscattered propagation. The light emitted from the POCAM can be detected by any IceCube DOM. 


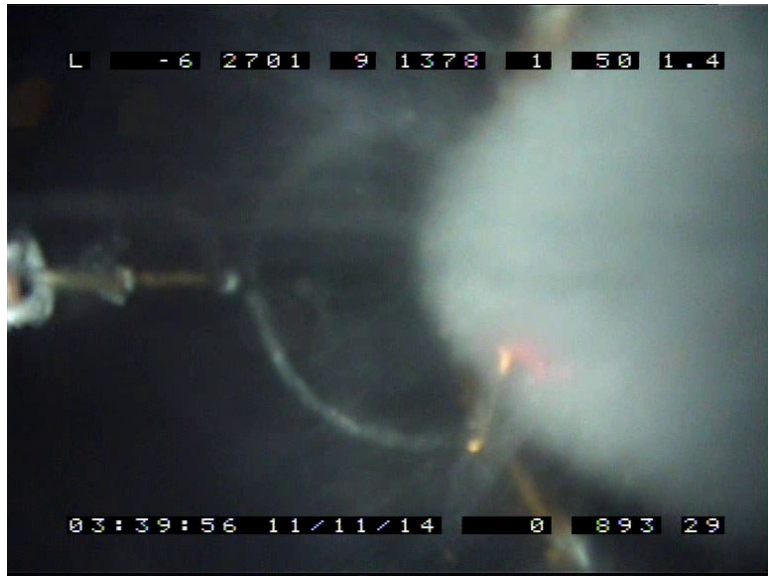

(a) Camera image downward looking into the hole ice

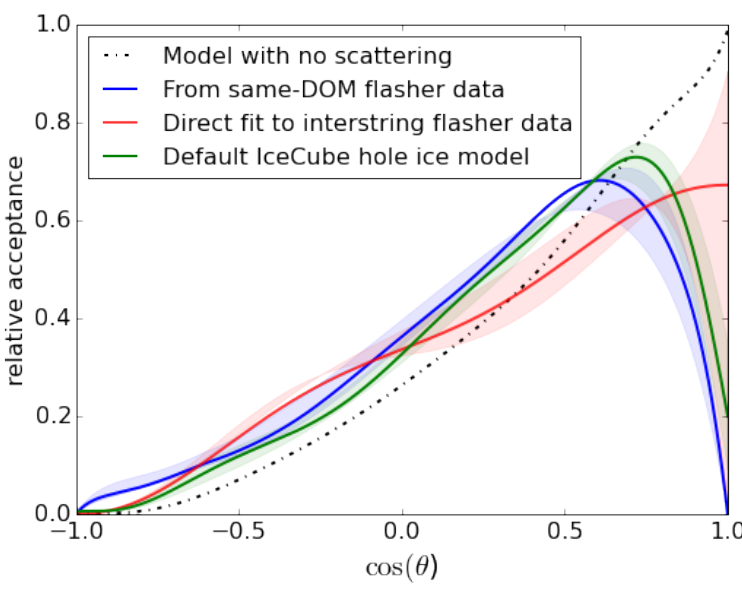

(b) DOM angular acceptance curves

Figure 5: Current understanding of the impact of the refrozen ice within the drilled holes on the DOM's angular acceptance.

Two kinds of datasets have been simulated. The reference dataset accounts for bulk ice propagation but no propagation through the hole ice and assumes the DOMs to be able to measure photons over their entire spherical surface. All other datasets assume a given realization of the hole ice and only allow photon detection at the PMT surface, representing the data from a potential measurement.

The DOM-wise ratio of detected photons in the data-like set and the reference dataset is equivalent to the DOMs in-situ angular acceptance function at the zenith angle of this DOM relative to the POCAM. By plotting the relative acceptance of many DOMs the overall angular acceptance of an average DOM is obtained. Figure 6a shows an example where the entire drill hole is assumed to be filled with a weakly scattering medium and all DOMs are located in the center of their respective hole. The obtained angular acceptance curve agrees well with the "Default IceCube hole ice model" which is based on the same assumptions. Figure 6b shows an example for a small, but strongly scattering central hole ice column. In addition the DOMs are now randomly positioned inside the drill hole. Obviously no consistent average angular acceptance curve can be obtained, as different parts of the DOM surface are being shaded off relative to the emitter. Using Monte Carlo knowledge about the geometry, we can identify causally connected curves which belong to similar relative geometries. In the experiment the geometric ambiguity can be resolved by a multitude of POCAMs illuminating each DOM from different azimuthal angles.

Given a satisfactory measurement in Gen2 Phase-1, the individual angular acceptance curves can also be interpreted in terms of the underlying parameters (size and scattering length of the hole ice and position of the DOM). This allows to treat the hole ice via direct photon propagation instead of the effective description via the angular acceptance curve, which neglects azimuthal effects.

\section{Summary and Outlook}

We have presented the concept and design of an isotropic, multi-wavelength and pulsed light source 


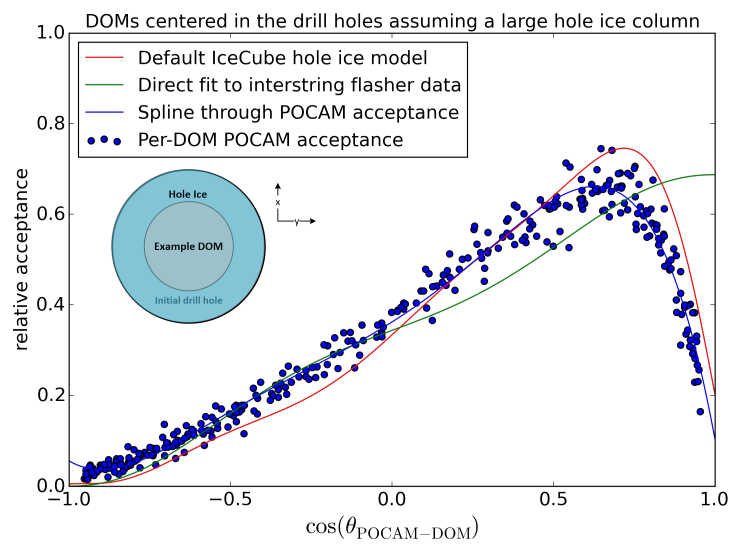

(a) Assuming the entire drill hole to be filled with a weakly scattering medium and the DOMs to be centered inside the holes (IceCube default)

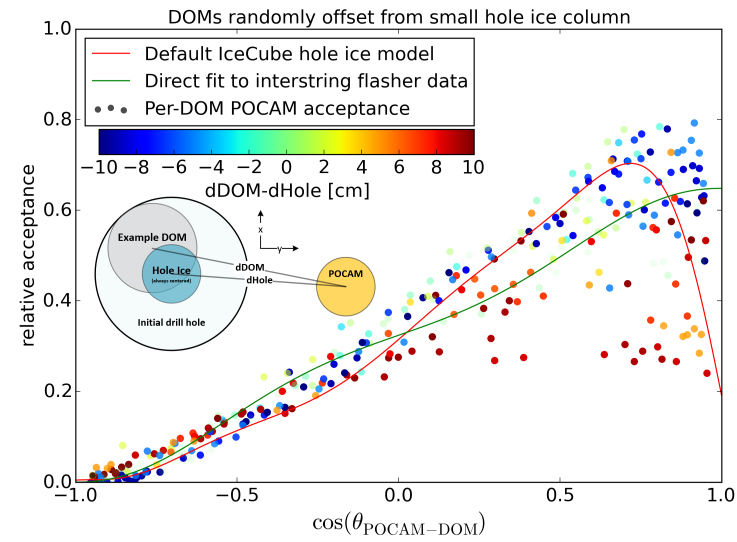

(b) Assuming a central, small and strongly scattering column with the DOMs randomly positioned inside the holes

Figure 6: POCAM capability for measuring the IceCube drill hole properties. The points represent different DOMs measuring light from a single POCAM at different angles.

to be used as a calibration device in IceCube-Gen2. In the baseline plan for IceCube-Gen2 Phase1, two POCAM modules on each string will enable us to determine in situ the relative optical efficiency and hole ice properties of the nearby DOMs. In addition the POCAMs will be used to re-evaluate bulk ice properties within the Gen2 Phase-1 volume, which can also be applied to the rest of the detector. The deployment of the first POCAM prototype in Baikal-GVD during 2017 permits the validation of the POCAM concept within a neutrino telescope and in the same time provides valuable information for GVD.

\section{Acknowledgments}

We thank the Baikal collaboration for allowing the deployment of the POCAM prototype in GVD.

\section{References}

[1] IceCube Collaboration, M. G. Aartsen et al., Nucl. Instrum. Meth. A711 (2013) 73-89.

[2] IceCube Collaboration, M. G. Aartsen et al., EPJ Web of Conferences 116 (2016) 06011.

[3] IceCube-Gen2 Collaboration, K. Krings, PoS ( ICRC2015) 1133 (2016).

[4] IceCube Gen2 Collaboration, M. Jurkovic et al., EPJ Web of Conferences 116 (2016) 06001.

[5] J. Kapustinsky et al., Nucl. Instr. Meth. Phys. Res. Sec.A 241 (1985) 612 - 613.

[6] A. D. Avrorin et al., PoS (EPS-HEP2015) 418 (2015).

[7] I. Belolaptikov et al., International Cosmic Ray Conference 1 (1995) 1043.

[8] V. A. Balkanov et al., Appl. Opt. 33 (1999) 6818.

[9] IceCube Collaboration, D. Chirkin, arXiv:1309.7010.

[10] IceCube Collaboration Collaboration, M. G. Aartsen et al., Phys. Rev. D 91 (Apr, 2015) 072004.

[11] IceCube Collaboration, R. Abbasi et al., Astropart. Phys. 35 (2012) 615-624. 